\title{
AN EFFECTIVE UNIVERSALITY THEOREM FOR THE RIEMANN ZETA FUNCTION
}

\author{
YOUNESS LAMZOURI, STEPHEN LESTER, AND MAKSYM RADZIWILL
}

\begin{abstract}
Let $0<r<1 / 4$, and $f$ be a non-vanishing continuous function in $|z| \leq r$, that is analytic in the interior. Voronin's universality theorem asserts that translates of the Riemann zeta function $\zeta(3 / 4+z+i t)$ can approximate $f$ uniformly in $|z|<r$ to any given precision $\varepsilon$, and moreover that the set of such $t \in[0, T]$ has measure at least $c(\varepsilon) T$ for some $c(\varepsilon)>0$, once $T$ is large enough. This was refined by Bagchi who showed that the measure of such $t \in[0, T]$ is $(c(\varepsilon)+o(1)) T$, for all but at most countably many $\varepsilon>0$. Using a completely different approach, we obtain the first effective version of Voronin's Theorem, by showing that in the rate of convergence one can save a small power of the logarithm of $T$. Our method is flexible, and can be generalized to other $L$-functions in the $t$-aspect, as well as to families of $L$-functions in the conductor aspect.
\end{abstract}

\section{InTRODUCTION}

In 1914 Fekete constructed a formal power series $\sum_{n=1}^{\infty} a_{n} x^{n}$ with the following universal property: For any continuous function $f$ on $[-1,1]$ (with $f(0)=0$ ) and given any $\varepsilon>0$ there exists an integer $N>0$ such that

$$
\sup _{-1 \leq x \leq 1}\left|\sum_{n \leq N} a_{n} x^{n}-f(x)\right|<\varepsilon .
$$

In the 1970's Voronin [14] discovered the remarkable fact that the Riemann zetafunction satisfies a similar universal property. He showed that for any $r<\frac{1}{4}$, any non-vanishing continuous function $f$ in $|z| \leq r$, which is analytic in the interior, and for arbitrary $\varepsilon>0$, there exists a $T>0$ such that

$$
\max _{|z| \leq r}\left|\zeta\left(\frac{3}{4}+i T+z\right)-f(z)\right|<\varepsilon .
$$

Voronin obtained a more quantitative description of this phenomena, stated below.

Voronin's universality theorem. Let $0<r<\frac{1}{4}$ be a real number. Let $f$ be a nonvanishing continuous function in $|z| \leq r$, that is analytic in the interior. Then, for any $\varepsilon>0$

$$
\liminf _{T \rightarrow \infty} \frac{1}{T} \cdot \operatorname{meas}\left\{T \leq t \leq 2 T: \max _{|z| \leq r}\left|\zeta\left(\frac{3}{4}+i t+z\right)-f(z)\right|<\varepsilon\right\}>0,
$$

2010 Mathematics Subject Classification. Primary 11M06.

The first and third authors are partially supported by Discovery Grants from the Natural Sciences and Engineering Research Council of Canada. 
where meas is Lebesgue's measure on $\mathbb{R}$.

There are several extensions of this theorem, for example to domains more general than compact discs (such as any compact set $K$ contained in the strip $1 / 2<\operatorname{Re}(s)<$ 1 and with connected complement), or to more general $L$-functions. For a complete history of this subject, we refer the reader to [11].

The assumption that $f(z) \neq 0$ is necessary: if $f$ were allowed to vanish then an application of Rouche's theorem would produce at least $\asymp T$ zeros $\rho=\beta+i \gamma$ of $\zeta(s)$ with $\beta>\frac{1}{2}+\varepsilon$ and $T \leq \gamma \leq 2 T$, contradicting the simplest zero-density theorems.

Subsequent work of Bagchi [1] clarified Voronin's universality theorem by setting it in the context of probability theory (see [7] for a streamlined proof). Viewing $\zeta\left(\frac{3}{4}+i t+z\right)$ with $t \in[T, 2 T]$ as a random variable $X_{T}$ in the space of random analytic functions (i.e $X_{T}(z)=\zeta\left(\frac{3}{4}+i U_{T}+z\right)$ with $U_{T}$ uniformly distributed in $\left.[T, 2 T]\right)$, Bagchi showed that as $T \rightarrow \infty$ this sequence of random variables converges in law (in the space of random analytic functions) to a random Euler product,

$$
\zeta(s, X):=\prod_{p}\left(1-\frac{X(p)}{p^{s}}\right)^{-1}
$$

with $\{X(p)\}_{p}$ a sequence of independent random variables uniformly distributed on the unit circle (and with $p$ running over prime numbers). This product converges almost surely for $\operatorname{Re}(s)>\frac{1}{2}$ and defines almost surely a holomorphic function in the half-plane $\operatorname{Re}(s)>\sigma_{0}$ for any $\sigma_{0}>\frac{1}{2}$ (see Section 2 below). The proof of Voronin's universality is then reduced to showing that the support of $\zeta(s+3 / 4, X)$ in the space of random analytic functions contains all non-vanishing analytic $f:\{z:|z|<r\} \rightarrow \mathbb{C} \backslash\{0\}$. Moreover it follows from Bagchi's work that the limit in Voronin's universality theorem exists for all but at most countably many $\varepsilon>0$.

In this paper, we present an alternative approach to Bagchi's result using methods from hard analysis. As a result we obtain, for the first time, a rate of convergence in Voronin's universality theorem. We also give an explicit description for the limit in terms of the random model $\zeta(s, X)$.

Theorem 1.1. Let $0<r<\frac{1}{4}$. Let $f$ be a non-vanishing continuous function on $|z| \leq(r+1 / 4) / 2$ that is holomorphic in $|z|<(r+1 / 4) / 2$. Let $\omega$ be a real-valued continuously differentiable function with compact support. Then, we have

$$
\begin{aligned}
\frac{1}{T} \int_{T}^{2 T} \omega\left(\max _{|z| \leq r}\left|\zeta\left(\frac{3}{4}+i t+z\right)-f(z)\right|\right) d t & =\mathbb{E}\left(\omega\left(\max _{|z| \leq r}\left|\zeta\left(\frac{3}{4}+z, X\right)-f(z)\right|\right)\right) \\
& +O\left((\log T)^{-\frac{(3 / 4-r)}{11}+o(1)}\right),
\end{aligned}
$$

where the constant in the $O$ depends on $f, \omega$ and $r$. 
If the random variable $Y_{r, f}=\max _{|z| \leq r}\left|\zeta\left(\frac{3}{4}+z, X\right)-f(z)\right|$ is absolutely continuous, then it follows from the proof of Theorem 1.1 that for any fixed $\varepsilon>0$ we have

$$
\begin{aligned}
& \frac{1}{T} \cdot \operatorname{meas}\left\{T \leq t \leq 2 T: \max _{|z| \leq r}\left|\zeta\left(\frac{3}{4}+i t+z\right)-f(z)\right|<\varepsilon\right\} \\
& =\mathbb{P}\left(\max _{|z| \leq r}\left|\zeta\left(\frac{3}{4}+z, X\right)-f(z)\right|<\varepsilon\right)+O\left((\log T)^{-\frac{(3 / 4-r)}{11}+o(1)}\right) .
\end{aligned}
$$

Unfortunately, we have not been able to even show that $Y_{r, f}$ has no jump discontinuities. We conjecture the latter to be true, and one might even hope that $Y_{r, f}$ is absolutely continuous.

A slight modification of the proof of Theorem 1.1 allows for more general domains than the disc $|z| \leq r$. Furthermore, if $\omega \geq \mathbf{1}_{(0, \varepsilon)}$ (where $\mathbf{1}_{S}$ is the indicator function of the set $S$ ), then it follows from Voronin's universality theorem that the main term in Theorem 1.1 is positive. Explicit lower bounds for the limit in (1.2) (in terms of $\varepsilon$ ) are contained in the papers of Good [3] and Garunkstis [2].

Our approach is flexible, and can be generalized to other $L$-functions in the $t$-aspect, as well as to "natural" families of $L$-functions in the conductor aspect. The only analytic ingredients that are needed are zero density estimates, and bounds on the coefficients of these $L$-functions (the so-called Ramanujan conjecture). In particular, the techniques of this paper can be used to obtain an effective version of a recent result of Kowalski [7], who proved an analogue of Voronin's universality theorem for families of $L$-functions attached to $G L_{2}$ automorphic forms. In fact, using the zero-density estimates near 1 that are known for a very large class of $L$-functions (including those in the Selberg class by Kaczorowski and Perelli [6], and for families of $L$-functions attached to $G L_{n}$ automorphic forms by Kowalski and Michel [8]), one can prove an analogue of Theorem 1.1 for these $L$-functions, where we replace $3 / 4$ by some $\sigma<1$ (and $r<1-\sigma$ ).

The main idea in the proof of Theorem 1.1 is to cover the boundary of the disc $|z| \leq r$ with a union of a growing (with $T$ ) number of discs, while maintaining a global control of the size of $\left|\zeta^{\prime}(s+z)\right|$ on $|z| \leq r$. It is enough to focus on the boundary of the disc thanks to the maximum modulus principle. The behavior of $\zeta(s+z)$ with $z$ localized to a shrinking disc is essentially governed by the behavior at a single point $z=z_{i}$ in the disc. This allows us to reduce the problem to understanding the joint distribution of a growing number of shifts $\log \zeta\left(s+z_{i}\right)$ with the $z_{i}$ well-spaced, which can be understood by computing the moments of these shifts and using standard Fourier techniques.

It seems very difficult to obtain a rate of convergence which is better than logarithmic in Theorem 1.1. We have at present no understanding as to what the correct rate of convergence should be. 


\section{Key IngREDIEnts And DETAILED RESUlts}

We first begin with stating certain important properties of the random model $\zeta(s, X)$. Let $\{X(p)\}_{p}$ be a sequence of independent random variables uniformly distributed on the unit circle. Then we have

$$
-\log \left(1-\frac{X(p)}{p^{s}}\right)=\frac{X(p)}{p^{s}}+h_{X}(p, s)
$$

where the random series

$$
\sum_{p} h_{X}(p, s)
$$

converges absolutely and surely for $\operatorname{Re}(s)>1 / 2$. Hence, it (almost surely) defines a holomorphic function in $s$ in this half-plane. Moreover, since $\mathbb{E}(X(p))=0$ and $\mathbb{E}\left(|X(p)|^{2}\right)=1$, then it follows from Kolmogorov's three-series theorem that the series

$$
\sum_{p} \frac{X(p)}{p^{s}}
$$

is almost surely convergent for $\operatorname{Re}(s)>1 / 2$. By well-known results on Dirichlet series, this shows that this series defines (almost surely) a holomorphic function on the halfplane $\operatorname{Re}(s)>\sigma_{0}$, for any $\sigma_{0}>1 / 2$. Thus, by taking the exponential of the sum of the random series in (2.1) and (2.2), it follows that $\zeta(s, X)$ converges almost surely to a holomorphic function on the half-plane $\operatorname{Re}(s)>\sigma_{0}$, for any $\sigma_{0}>1 / 2$.

We extend the $X(p)$ multiplicatively to all positive integers by setting $X(1)=1$ and $X(n):=X\left(p_{1}\right)^{a_{1}} \cdots X\left(p_{k}\right)^{a_{k}}$, if $n=p_{1}^{a_{1}} \ldots p_{k}^{a_{k}}$. Then we have

$$
\mathbb{E}(X(n) \overline{X(m)})= \begin{cases}1 & \text { if } m=n \\ 0 & \text { otherwise }\end{cases}
$$

Furthermore, for any complex number $s$ with $\operatorname{Re}(s)>1 / 2$ we have almost surely that

$$
\zeta(s, X)=\sum_{n=1}^{\infty} \frac{X(n)}{n^{s}} .
$$

To compare the distribution of $\zeta(s+i t)$ to that of $\zeta(s, X)$, we define a probability measure on $[T, 2 T]$ in a standard way, by

$$
\mathbb{P}_{T}(S):=\frac{1}{T} \operatorname{meas}(S), \text { for any } S \subseteq[T, 2 T] .
$$

The idea behind our proof of effective universality is to first reduce the problem to the discrete problem of controlling the distribution of many shifts $\log \zeta\left(s_{j}+i t\right)$ with all of the $s_{j}$ contained in a compact set inside the strip $\frac{1}{2}<\operatorname{Re}(s)<1$. One of the main ingredients in this reduction is the following result which allows us to control the maximum of the derivative of the Riemann zeta-function. This is proven in Section 4 . 
Proposition 2.1. Let $0<r<1 / 4$ be fixed. Then there exist positive constants $b_{1}, b_{2}$ and $b_{3}$ (that depend only on $r$ ) such that

$$
\mathbb{P}_{T}\left(\max _{|z| \leq r}\left|\zeta^{\prime}\left(\frac{3}{4}+i t+z\right)\right|>e^{V}\right) \ll \exp \left(-b_{1} V^{\frac{1}{1-\sigma(r)}}(\log V)^{\frac{\sigma(r)}{1-\sigma(r)}}\right)
$$

where $\sigma(r)=\frac{3}{4}-r$, uniformly for $V$ in the range $b_{2}<V \leq b_{3}(\log T)^{1-\sigma} /(\log \log T)$.

We also prove an analogous result for the random model $\zeta(s, X)$, which holds for all sufficiently large $V$.

Proposition 2.2. Let $0<r<1 / 4$ be fixed and $\sigma(r)=\frac{3}{4}-r$. Then there exist positive constants $b_{1}$ and $b_{2}$ (that depend only on $r$ ) such that for all $V>b_{2}$ we have

$$
\mathbb{P}\left(\max _{|z| \leq r}\left|\zeta^{\prime}\left(\frac{3}{4}+z, X\right)\right|>e^{V}\right) \ll \exp \left(-b_{1} V^{\frac{1}{1-\sigma(r)}}(\log V)^{\frac{\sigma(r)}{1-\sigma(r)}}\right) .
$$

Once the reduction has been accomplished, it remains to understand the joint distribution of the shifts $\left\{\log \zeta\left(s_{1}+i t\right), \log \zeta\left(s_{2}+i t\right), \ldots, \log \zeta\left(s_{J}+i t\right)\right\}$ with $J \rightarrow \infty$ as $T \rightarrow \infty$ at a certain rate, and $s_{1}, \ldots, s_{J}$ are complex numbers with $\frac{1}{2}<\operatorname{Re}\left(s_{j}\right)<1$ for all $j \leq J$. Heuristically, this should be well approximated by the the joint distribution of the random variables $\left\{\log \zeta\left(s_{1}, X\right), \log \zeta\left(s_{2}, X\right), \ldots, \log \zeta\left(s_{J}, X\right)\right\}$. In order to establish this fact (in a certain range of $J$ ), we first prove, in Section 5, that the moments of the joint shifts $\log \zeta\left(s_{j}+i t\right)$ are very close to the corresponding ones of $\log \zeta\left(s_{j}, X\right)$, for $j \leq J$.

Theorem 2.1. Fix $1 / 2<\sigma_{0}<1$. Let $s_{1}, s_{2}, \ldots, s_{k}, r_{1}, \ldots, r_{\ell}$ be complex numbers in the rectangle $\sigma_{0}<\operatorname{Re}(z)<1$ and $|\operatorname{Im}(z)| \leq T^{\left(\sigma_{0}-1 / 2\right) / 4}$. Then, there exist positive constants $c_{3}, c_{4}, c_{5}$ and a set $\mathcal{E}(T) \subset[T, 2 T]$ of measure $\ll T^{1-c_{3}}$, such that if $k, \ell \leq$ $c_{4} \log T / \log \log T$ then

$$
\begin{aligned}
& \frac{1}{T} \int_{[T, 2 T] \backslash \mathcal{E}(T)}\left(\prod_{j=1}^{k} \log \zeta\left(s_{j}+i t\right)\right)\left(\prod_{j=1}^{\ell} \log \zeta\left(r_{j}-i t\right)\right) d t \\
& =\mathbb{E}\left(\left(\prod_{j=1}^{k} \log \zeta\left(s_{j}, X\right)\right)\left(\prod_{j=1}^{\ell} \log \overline{\zeta\left(r_{j}, X\right)}\right)\right)+O\left(T^{-c_{5}}\right) .
\end{aligned}
$$

Having obtained the moments we are in position to understand the characteristic function,

$$
\Phi_{T}(\mathbf{u}, \mathbf{v}):=\frac{1}{T} \int_{T}^{2 T} \exp \left(i\left(\sum_{j=1}^{J}\left(u_{j} \operatorname{Re} \log \zeta\left(s_{j}+i t\right)+v_{j} \operatorname{Im} \log \zeta\left(s_{j}+i t\right)\right)\right)\right) d t
$$

where $\mathbf{u}=\left(u_{1}, \ldots, u_{J}\right) \in \mathbb{R}^{J}$ and $\mathbf{v}=\left(v_{1}, \ldots, v_{J}\right) \in \mathbb{R}^{J}$. We relate the above characteristic function to the characteristic function of the probabilistic model,

$$
\Phi_{\text {rand }}(\mathbf{u}, \mathbf{v}):=\mathbb{E}\left(\exp \left(i\left(\sum_{j=1}^{J}\left(u_{j} \operatorname{Re} \log \zeta\left(s_{j}, X\right)+v_{j} \operatorname{Im} \log \zeta\left(s_{j}, X\right)\right)\right)\right)\right) .
$$


This is obtained in the following theorem, which we prove in Section 6.

Theorem 2.2. Fix $1 / 2<\sigma<1$. Let $T$ be large and $J \leq(\log T)^{\sigma}$ be a positive integer. Let $s_{1}, s_{2}, \ldots, s_{J}$ be complex numbers such that $\min \left(\operatorname{Re}\left(s_{j}\right)\right)=\sigma$ and $\max \left(\left|\operatorname{Im}\left(s_{j}\right)\right|\right)<$ $T^{(\sigma-1 / 2) / 4}$. Then, there exist positive constants $c_{1}(\sigma), c_{2}(\sigma)$, such that for all $\mathbf{u}, \mathbf{v} \in \mathbb{R}^{J}$ such that $\max \left(\left|u_{j}\right|\right), \max \left(\left|v_{j}\right|\right) \leq c_{1}(\sigma)(\log T)^{\sigma} / J$ we have

$$
\Phi_{T}(\mathbf{u}, \mathbf{v})=\Phi_{\text {rand }}(\mathbf{u}, \mathbf{v})+O\left(\exp \left(-c_{2}(\sigma) \frac{\log T}{\log \log T}\right)\right) .
$$

Using this result, we can show that the joint distribution of the shifts $\log \zeta\left(s_{j}+i t\right)$ is very close to the corresponding joint distribution of the random variables $\log \zeta\left(s_{j}, X\right)$. The proof depends on Beurling-Selberg functions. To measure how close are these distributions, we introduce the discrepancy $\mathcal{D}_{T}\left(s_{1}, \ldots, s_{J}\right)$ defined as

$$
\sup _{\left(\mathcal{R}_{1}, \ldots, \mathcal{R}_{J}\right) \subset \mathbb{C}^{J}}\left|\mathbb{P}_{T}\left(\log \zeta\left(s_{j}+i t\right) \in \mathcal{R}_{j}, \forall j \leq J\right)-\mathbb{P}\left(\log \zeta\left(s_{j}, X\right) \in \mathcal{R}_{j}, \forall j \leq J\right)\right|
$$

where the supremum is taken over all $\left(\mathcal{R}_{1}, \ldots, \mathcal{R}_{J}\right) \subset \mathbb{C}^{J}$ and for each $j=1, \ldots, J$ the set $\mathcal{R}_{j}$ is a rectangle with sides parallel to the coordinate axes. Our next theorem, proven in Section [7, states a bound for the above discrepancy. This generalizes Theorem 1.1 of [10], which corresponds to the special case $J=1$.

Theorem 2.3. Let $T$ be large, $\frac{1}{2}<\sigma<1$ and $J \leq(\log T)^{\sigma / 2}$ be a positive integer. Let $s_{1}, s_{2}, \ldots, s_{J}$ be complex numbers such that

$$
\frac{1}{2}<\sigma:=\min _{j}\left(\operatorname{Re}\left(s_{j}\right)\right) \leq \max _{j}\left(\operatorname{Re}\left(s_{j}\right)\right)<1 \quad \text { and } \quad \max _{j}\left(\left|\operatorname{Im}\left(s_{j}\right)\right|\right)<T^{\left(\sigma-\frac{1}{2}\right) / 4} .
$$

Then, we have

$$
\mathcal{D}_{T}\left(s_{1}, \ldots, s_{J}\right) \ll \frac{J^{2}}{(\log T)^{\sigma}} .
$$

With all of the above tools in place we are ready to prove Theorem 1.1. This is accomplished in the next section.

\section{Effective universality: Proof of Theorem 1.1}

In this section, we will prove Theorem 1.1 using the results described in Section 2 . First, by the maximum modulus principle, the maximum of $\left|\zeta\left(\frac{3}{4}+i t+z\right)-f(z)\right|$ in the disc $\{z:|z| \leq r\}$ must occur on its boundary $\{z:|z|=r\}$. Our idea consists

of first covering the circle $|z|=r$ with $J$ discs of radius $\varepsilon$ and centres $z_{j}$, where $z_{j} \in\{z:|z|=r\}$ for all $1 \leq j \leq J$, and $J \asymp 1 / \varepsilon$. We call each of the discs $\mathcal{D}_{j}$. Then, we observe that

$$
\max _{j \leq J}\left|\zeta\left(\frac{3}{4}+i t+z_{j}\right)-f\left(z_{j}\right)\right| \leq \max _{|z| \leq r}\left|\zeta\left(\frac{3}{4}+i t+z\right)-f(z)\right| \leq \max _{j \leq J} \max _{z \in \mathcal{D}_{j}}\left|\zeta\left(\frac{3}{4}+i t+z\right)-f(z)\right| .
$$


Using Proposition 2.1, we shall prove that for all $j \leq J$ (where $J$ is a small power of $\log T$ ) we have

$$
\max _{z \in \mathcal{D}_{j}}\left|\zeta\left(\frac{3}{4}+i t+z\right)-f(z)\right| \approx\left|\zeta\left(\frac{3}{4}+i t+z_{j}\right)-f\left(z_{j}\right)\right|
$$

for all $t \in[T, 2 T]$ except for a set of points $t$ of very small measure. We will then deduce that the (weighted) distribution of $\max _{|z| \leq r}\left|\zeta\left(\frac{3}{4}+i t+z\right)-f(z)\right|$ is very close to the corresponding distribution of $\max _{j \leq J}\left|\zeta\left(\frac{3}{4}+i t+z_{j}\right)-f\left(z_{j}\right)\right|$, for $t \in[T, 2 T]$. We will also establish an analogous result for the random model $\zeta(s, X)$ along the same lines, by using Proposition 2.2 instead of Proposition 2.1. Therefore, to complete the proof of Theorem 1.1 we need to compare the distributions of $\max _{j \leq J}\left|\zeta\left(\frac{3}{4}+i t+z_{j}\right)-f\left(z_{j}\right)\right|$ and $\max _{j \leq J}\left|\zeta\left(\frac{3}{4}+z_{j}, X\right)-f\left(z_{j}\right)\right|$. Using Theorem 2.3 we prove

Proposition 3.1. Let $T$ be large, $0<r<1 / 4$ and $J \leq(\log T)^{(3 / 4-r) / 7}$ be a positive integer. Let $z_{1}, \ldots, z_{J}$ be complex numbers such that $\left|z_{j}\right| \leq r$. Then we have

$$
\begin{aligned}
& \left|\mathbb{P}_{T}\left(\max _{j \leq J}\left|\zeta\left(\frac{3}{4}+i t+z_{j}\right)-f\left(z_{j}\right)\right| \leq u\right)-\mathbb{P}\left(\max _{j \leq J}\left|\zeta\left(\frac{3}{4}+z_{j}, X\right)-f\left(z_{j}\right)\right| \leq u\right)\right| \\
& \ll_{u} \frac{(J \log \log T)^{6 / 5}}{(\log T)^{(3 / 4-r) / 5}} .
\end{aligned}
$$

Proof. Fix a positive real number $u$. Let $\mathcal{A}_{J}(T)$ be the set of those $t$ for which $\mid \arg \zeta\left(\frac{3}{4}+\right.$ $\left.i t+z_{j}\right) \mid \leq \log \log T$ for every $j \leq J$. Since $\operatorname{Re}\left(\frac{3}{4}+i t+z_{j}\right) \geq \frac{3}{4}-r$ and $\operatorname{Im}\left(\frac{3}{4}+i t+z_{j}\right)=$ $t+O(1)$, then it follows from Theorem 1.1 and Remark 1 of [9] that for each $j \leq J$ we have

$$
\mathbb{P}_{T}\left(\left|\arg \zeta\left(\frac{3}{4}+i t+z_{j}\right)\right| \geq \log \log T\right) \ll \exp \left(-(\log \log T)^{\left(\frac{1}{4}+r\right)^{-1}}\right) \ll \frac{1}{(\log T)^{4}}
$$

Therefore, we obtain

$\mathbb{P}_{T}\left([T, 2 T] \backslash \mathcal{A}_{J}(T)\right) \leq \sum_{j=1}^{J} \mathbb{P}_{T}\left(\left|\arg \zeta\left(\frac{3}{4}+i t+z_{j}\right)\right| \geq \log \log T\right) \ll \frac{J}{(\log T)^{4}} \ll \frac{1}{(\log T)^{2}}$,

and this implies that

$$
\begin{aligned}
\mathbb{P}_{T}\left(\max _{j \leq J}\left|\zeta\left(\frac{3}{4}+i t+z_{j}\right)-f\left(z_{j}\right)\right| \leq u\right) & =\mathbb{P}_{T}\left(\max _{j \leq J}\left|\zeta\left(\frac{3}{4}+i t+z_{j}\right)-f\left(z_{j}\right)\right| \leq u, t \in \mathcal{A}_{J}(T)\right) \\
& +O\left(\frac{1}{(\log T)^{2}}\right) .
\end{aligned}
$$

For each $j \leq J$ consider the region

$$
\mathcal{U}_{j}=\left\{z:\left|e^{z}-f\left(z_{j}\right)\right| \leq u,|\operatorname{Im}(z)| \leq \log \log T\right\} .
$$

We cover $\mathcal{U}_{j}$ with $K \asymp \operatorname{area}\left(\mathcal{U}_{j}\right) / \varepsilon^{2} \asymp \log \log T / \varepsilon^{2}$ squares $\mathcal{R}_{j, k}$ with sides of length $\varepsilon=\varepsilon(T)$, where $\varepsilon$ is a small positive parameter to be chosen later. Let $\mathcal{K}_{j}$ denote the set of $k \in\{1,2, \ldots, K\}$ such that the intersection of $\mathcal{R}_{j, k}$ with the boundary of $\mathcal{U}_{j}$ is 
empty and write $\mathcal{K}_{j}^{c}$ for the relative complement of $\mathcal{K}_{j}$ with respect to $\{1,2, \ldots, K\}$. Note that $\left|\mathcal{K}_{j}^{c}\right| \asymp \log \log T / \varepsilon$. By construction,

$$
\left(\bigcup_{k \in \mathcal{K}_{j}} \mathcal{R}_{j, k}\right) \subset \mathcal{U}_{j} \subset\left(\bigcup_{k \leq K} \mathcal{R}_{j, k}\right) .
$$

Therefore (3.3) can be expressed as

$$
\mathbb{P}_{T}\left(\forall j \leq J, \forall k \leq K: \log \zeta\left(\frac{3}{4}+i t+z_{j}\right) \in \mathcal{R}_{j, k}\right)+\mathcal{E}_{1}
$$

where by Theorem 2.3

$$
\begin{aligned}
\mathcal{E}_{1} & \ll \sum_{j \leq J} \sum_{k \in \mathcal{K}_{j}^{c}} \mathbb{P}_{T}\left(\log \zeta\left(\frac{3}{4}+i t+z_{j}\right) \in \mathcal{R}_{j, k}\right) \\
& \ll \sum_{j \leq J} \sum_{k \in \mathcal{K}_{j}^{c}}\left(\mathbb{P}_{T}\left(\log \zeta\left(\frac{3}{4}+z_{j}, X\right) \in \mathcal{R}_{j, k}\right)+\frac{1}{(\log T)^{3 / 4-r}}\right) \\
& \ll J \cdot \frac{\log \log T}{\varepsilon}\left(\varepsilon^{2}+\frac{1}{(\log T)^{3 / 4-r}}\right)
\end{aligned}
$$

and in the last step we used the fact that $\log \zeta(s, X)$ is an absolutely continuous random variable (see for example Jessen and Wintner [5]). We conclude that

$$
\begin{aligned}
\mathbb{P}_{T}\left(\max _{j \leq J}\left|\zeta\left(\frac{3}{4}+i t+z_{j}\right)-f\left(z_{j}\right)\right| \leq u\right) & =\mathbb{P}_{T}\left(\forall j \leq J, \forall k \leq K: \log \zeta\left(\frac{3}{4}+i t+z_{j}\right) \in \mathcal{R}_{j, k}\right) \\
& +O\left(\varepsilon J \log \log T+\frac{J \log \log T}{\varepsilon(\log T)^{3 / 4-r}}\right) .
\end{aligned}
$$

Additionally, it follows from Theorem 2.3 that the main term of this last estimate equals

$$
\mathbb{P}\left(\forall j \leq J, \forall k \leq K: \log \zeta\left(\frac{3}{4}+z_{j}, X\right) \in \mathcal{R}_{j, k}\right)+O\left(\frac{J^{2}(\log \log T)^{2}}{\varepsilon^{4}(\log T)^{3 / 4-r}}\right)
$$

We now repeat the exact same argument but for the random model $\zeta(s, X)$ instead of the zeta function. In particular, instead of (3.2) we shall use that

$$
\mathbb{P}\left(\left|\arg \zeta\left(\frac{3}{4}+z_{j}, X\right)\right| \geq \log \log T\right) \ll \exp \left(-(\log \log T)^{\left(\frac{1}{4}+r\right)^{-1}}\right) \ll \frac{1}{(\log T)^{4}}
$$

which follows from Theorem 1.9 of $[9$. Thus, similarly to (3.5) we obtain

$$
\begin{aligned}
\mathbb{P}\left(\forall j \leq J, \forall k \leq K: \log \zeta\left(\frac{3}{4}+z_{j}, X\right) \in \mathcal{R}_{j, k}\right) & =\mathbb{P}\left(\max _{j \leq J}\left|\zeta\left(\frac{3}{4}+z_{j}, X\right)-f\left(z_{j}\right)\right| \leq u\right) \\
& +O\left(\varepsilon J \log \log T+\frac{J \log \log T}{\varepsilon(\log T)^{3 / 4-r}}\right)
\end{aligned}
$$


Combining the above estimate with (3.5) and (3.6) we conclude that

$$
\begin{aligned}
\mathbb{P}_{T}\left(\max _{j \leq J}\left|\zeta\left(\frac{3}{4}+i t+z_{j}\right)-f\left(z_{j}\right)\right| \leq u\right) & =\mathbb{P}\left(\max _{j \leq J}\left|\zeta\left(\frac{3}{4}+z_{j}, X\right)-f\left(z_{j}\right)\right| \leq u\right) \\
& +O\left(\frac{J^{2}(\log \log T)^{2}}{\varepsilon^{4}(\log T)^{3 / 4-r}}+\varepsilon J \log \log T\right) .
\end{aligned}
$$

Finally, choosing

$$
\varepsilon=\left(\frac{J \log \log T}{(\log T)^{3 / 4-r}}\right)^{1 / 5}
$$

completes the proof.

Proof of Theorem 1.1. We wish to estimate

$$
\frac{1}{T} \int_{T}^{2 T} \omega\left(\max _{|z| \leq r}\left|\zeta\left(\frac{3}{4}+i t+z\right)-f(z)\right|\right) d t
$$

with $f$ an analytic non-vanishing function, and where $\omega$ is a continuously differentiable function with compact support.

Recall that the maximum of $\left|\zeta\left(\frac{3}{4}+i t+z\right)-f(z)\right|$ on the disc $\{z:|z| \leq r\}$ must occur on its boundary $\{z:|z|=r\}$, by the maximum modulus principle. Let $\varepsilon \leq(1 / 4-r) / 4$ be a small positive parameter to be chosen later, and cover the circle $|z|=r$ with $J \asymp 1 / \varepsilon \operatorname{discs} \mathcal{D}_{j}$ of radius $\varepsilon$ and centres $z_{j}$, where $z_{j} \in\{z:|z|=r\}$ for all $j \leq J$.

Let $\mathcal{S}_{V}(T)$ denote the set of those $t \in[T, 2 T]$ such that

$$
\max _{|z| \leq(r+1 / 4) / 2}\left|\zeta^{\prime}\left(\frac{3}{4}+i t+z\right)\right| \leq e^{V}
$$

where $V \leq \log \log T$ is a large parameter to be chosen later, and let $L:=\max _{|z| \leq(r+1 / 4) / 2}\left|f^{\prime}(z)\right|$. Then for $t \in \mathcal{S}_{V}(T)$, and for all $z \in \mathcal{D}_{j}$ we have

$$
\begin{aligned}
& \left|\zeta\left(\frac{3}{4}+i t+z\right)-f(z)-\left(\zeta\left(\frac{3}{4}+i t+z_{j}\right)-f\left(z_{j}\right)\right)\right|=\left|\int_{z_{j}}^{z} \zeta^{\prime}\left(\frac{3}{4}+i t+s\right)-f^{\prime}(s) d s\right| \\
& \leq\left|z-z_{j}\right| \cdot\left(\max _{|z| \leq(r+1 / 4) / 2}\left|\zeta^{\prime}\left(\frac{3}{4}+i t+z\right)\right|+L\right) \leq \varepsilon\left(e^{V}+L\right) \leq C \varepsilon e^{V},
\end{aligned}
$$

for some large absolute constant $C$, depending at most on $L$. Define

$$
\theta(t):=\max _{|z| \leq r}\left|\zeta\left(\frac{3}{4}+i t+z\right)-f(z)\right|-\max _{j \leq J}\left|\zeta\left(\frac{3}{4}+i t+z_{j}\right)-f\left(z_{j}\right)\right|
$$

Then, it follows from (3.1) and (3.8) that for all $t \in \mathcal{S}_{V}(T)$ we have

$$
0 \leq \theta(t) \leq C \varepsilon e^{V} .
$$


Therefore, using this estimate together with Proposition 2.1 and the fact that $\omega$ is bounded, we deduce that (3.7) equals

$$
\begin{aligned}
& \frac{1}{T} \int_{t \in \mathcal{S}_{V}(T)} \omega\left(\max _{j \leq J}\left|\zeta\left(\frac{3}{4}+i t+z_{j}\right)-f\left(z_{j}\right)\right|+\theta(t)\right) d t+O\left(e^{-V^{2}}\right) \\
& =\frac{1}{T} \int_{t \in \mathcal{S}_{V}(T)} \omega\left(\max _{j \leq J}\left|\zeta\left(\frac{3}{4}+i t+z_{j}\right)-f\left(z_{j}\right)\right|\right) d t+O\left(\left|\mathcal{E}_{2}\right|+e^{-V^{2}}\right) \\
& =\frac{1}{T} \int_{T}^{2 T} \omega\left(\max _{j \leq J}\left|\zeta\left(\frac{3}{4}+i t+z_{j}\right)-f\left(z_{j}\right)\right|\right) d t+O\left(\left|\mathcal{E}_{2}\right|+e^{-V^{2}}\right)
\end{aligned}
$$

where

$$
\mathcal{E}_{2}=\frac{1}{T} \int_{t \in \mathcal{S}_{V}(T)} \int_{0}^{\theta(t)} \omega^{\prime}\left(\max _{j \leq J}\left|\zeta\left(\frac{3}{4}+i t+z_{j}\right)-f\left(z_{j}\right)\right|+x\right) d x \cdot d t \ll \varepsilon e^{V},
$$

using the fact that $\omega^{\prime}$ is bounded on $\mathbb{R}$ together with (3.9).

Furthermore, observe that

$$
\begin{aligned}
& \frac{1}{T} \int_{T}^{2 T} \omega\left(\max _{j \leq J}\left|\zeta\left(\frac{3}{4}+i t+z_{j}\right)-f\left(z_{j}\right)\right|\right) d t \\
= & -\frac{1}{T} \int_{T}^{2 T} \int_{\max _{j \leq J}\left|\zeta\left(\frac{3}{4}+i t+z_{j}\right)-f\left(z_{j}\right)\right|}^{\infty} \omega^{\prime}(u) d u \cdot d t \\
= & -\int_{0}^{\infty} \omega^{\prime}(u) \cdot \mathbb{P}_{T}\left(\max _{j \leq J}\left|\zeta\left(\frac{3}{4}+i t+z_{j}\right)-f\left(z_{j}\right)\right| \leq u\right) d u .
\end{aligned}
$$

Since $\omega$ has a compact support, then $\omega^{\prime}(u)=0$ if $u>A$ for some positive constant $A$. Furthermore, it follows from Proposition 3.1 that for all $0 \leq u \leq A$ we have

$$
\begin{aligned}
\mathbb{P}_{T}\left(\max _{j \leq J}\left|\zeta\left(\frac{3}{4}+i t+z_{j}\right)-f\left(z_{j}\right)\right| \leq u\right) & =\mathbb{P}\left(\max _{j \leq J}\left|\zeta\left(\frac{3}{4}+z_{j}, X\right)-f\left(z_{j}\right)\right| \leq u\right) \\
& +O\left(\frac{(J \log \log T)^{6 / 5}}{(\log T)^{(3 / 4-r) / 5}}\right) .
\end{aligned}
$$

Inserting this estimate in (3.11) gives that

$$
\begin{aligned}
& \frac{1}{T} \int_{T}^{2 T} \omega\left(\max _{j \leq J}\left|\zeta\left(\frac{3}{4}+i t+z_{j}\right)-f\left(z_{j}\right)\right|\right) d t \\
& =-\int_{0}^{\infty} \omega^{\prime}(u) \cdot \mathbb{P}\left(\max _{j \leq J}\left|\zeta\left(\frac{3}{4}+z_{j}, X\right)-f\left(z_{j}\right)\right| \leq u\right) d u+O\left(\frac{(J \log \log T)^{6 / 5}}{(\log T)^{(3 / 4-r) / 5}}\right) \\
& =\mathbb{E}\left(\omega\left(\max _{j \leq J}\left|\zeta\left(\frac{3}{4}+z_{j}, X\right)-f\left(z_{j}\right)\right|\right)\right)+O\left(\frac{(J \log \log T)^{6 / 5}}{(\log T)^{(3 / 4-r) / 5}}\right) .
\end{aligned}
$$

To finish the proof, we shall appeal to the same argument used to establish (3.10), in order to compare the (weighted) distributions of $\max _{j \leq J}\left|\zeta\left(\frac{3}{4}+z_{j}, X\right)-f\left(z_{j}\right)\right|$ and $\max _{|z| \leq r}\left|\zeta\left(\frac{3}{4}+z, X\right)-f(z)\right|$. Let $\mathcal{S}_{V}(X)$ denote the event corresponding to

$$
\max _{|z| \leq(r+1 / 4) / 2}\left|\zeta^{\prime}\left(\frac{3}{4}+z, X\right)\right| \leq e^{V},
$$


and let $\mathcal{S}_{V}^{c}(X)$ be its complement. Then, it follows from Proposition 2.2 that $\mathbb{P}\left(\mathcal{S}_{V}^{c}(X)\right) \ll$ $\exp \left(-V^{2}\right)$. Moreover, similarly to (3.8) one can see that for all outcomes in $\mathcal{S}_{V}(X)$ we have, for all $z \in \mathcal{D}_{j}$

$$
\left|\zeta\left(\frac{3}{4}+z, X\right)-f(z)-\left(\zeta\left(\frac{3}{4}+z_{j}, X\right)-f\left(z_{j}\right)\right)\right|=\left|\int_{z_{j}}^{z} \zeta^{\prime}\left(\frac{3}{4}+s, X\right)-f^{\prime}(s) d s\right| \ll \varepsilon e^{V} .
$$

Thus, since the maximum of $\left|\zeta\left(\frac{3}{4}+z, X\right)-f(z)\right|$ for $|z| \leq r$ occurs (almost surely) on the boundary $|z|=r$, then following the argument leading to (3.10), we conclude that

$$
\begin{aligned}
& \mathbb{E}\left(\omega\left(\max _{|z| \leq r}\left|\zeta\left(\frac{3}{4}+z, X\right)-f(z)\right|\right)\right) \\
& =\mathbb{E}\left(\mathbf{1}_{\mathcal{S}_{V}(X)} \omega\left(\max _{|z| \leq r}\left|\zeta\left(\frac{3}{4}+z, X\right)-f(z)\right|\right)\right)+O\left(e^{-V^{2}}\right) \\
& =\mathbb{E}\left(\mathbf{1}_{\mathcal{S}_{V}(X)} \omega\left(\max _{j \leq J}\left|\zeta\left(\frac{3}{4}+z_{j}, X\right)-f(z)\right|\right)\right)+O\left(\varepsilon e^{V}+e^{-V^{2}}\right) \\
& =\mathbb{E}\left(\omega\left(\max _{j \leq J}\left|\zeta\left(\frac{3}{4}+z_{j}, X\right)-f(z)\right|\right)\right)+O\left(\varepsilon e^{V}+e^{-V^{2}}\right) .
\end{aligned}
$$

Finally, combining this estimate with (3.10) and (3.12), and noting that $J \asymp 1 / \varepsilon$ we deduce that

$$
\begin{aligned}
\frac{1}{T} \int_{T}^{2 T} \omega\left(\max _{|z| \leq r}\left|\zeta\left(\frac{3}{4}+i t+z\right)-f(z)\right|\right) d t & =\mathbb{E}\left(\omega\left(\max _{|z| \leq r}\left|\zeta\left(\frac{3}{4}+z, X\right)-f(z)\right|\right)\right) \\
& +O\left(\varepsilon e^{V}+e^{-V^{2}}+O\left(\frac{(\log \log T)^{6 / 5}}{\varepsilon^{6 / 5}(\log T)^{(3 / 4-r) / 5}}\right)\right) .
\end{aligned}
$$

Choosing $\varepsilon=(\log T)^{-(3 / 4-r) / 11}$ and $V=2 \sqrt{\log \log T}$ completes the proof.

\section{Controlling the Derivatives of the Zeta FunCtion And the Random} Model: Proof of Propositions 2.1 and 2.2

By Cauchy's theorem we have

$$
\left|\zeta^{\prime}\left(\frac{3}{4}+i t+z\right)\right| \leq \frac{1}{\delta} \max _{|s-z|=\delta}\left|\zeta\left(\frac{3}{4}+i t+s\right)\right|,
$$

and hence we get

$$
\max _{|z| \leq r}\left|\zeta^{\prime}\left(\frac{3}{4}+i t+z\right)\right| \leq \frac{1}{\delta} \max _{|s| \leq r+\delta}\left|\zeta\left(\frac{3}{4}+i t+s\right)\right| .
$$

Therefore, it follows that

$$
\begin{aligned}
\mathbb{P}_{T}\left(\max _{|z| \leq r}\left|\zeta^{\prime}\left(\frac{3}{4}+i t+z\right)\right|>e^{V}\right) & \leq \mathbb{P}_{T}\left(\max _{|s| \leq r+\delta}\left|\zeta\left(\frac{3}{4}+i t+s\right)\right|>\delta e^{V}\right) \\
& =\mathbb{P}_{T}\left(\max _{|s| \leq r+\delta} \log \left|\zeta\left(\frac{3}{4}+i t+s\right)\right|>V+\log \delta\right)
\end{aligned}
$$


To bound the RHS we estimate large moments of $\log \zeta\left(\frac{3}{4}+i t+s\right)$. This is accomplished by approximating $\log \zeta\left(\frac{3}{4}+i t+s\right)$ by a short Dirichlet polynomial, uniformly for all $s$ in the disc $\{|s| \leq r+\delta\}$. Using zero density estimates and large sieve inequalities, we can show that such an approximation holds for all $t \in[T, 2 T]$, except for an exceptional set of $t$ 's with very small measure. We prove

Lemma 4.1. Let $0<r<1 / 4$ be fixed, and $\delta=(1 / 4-r) / 4$. Let $y \leq \log T$ be a real number. There exists a set $\mathcal{I}(T) \subset[T, 2 T]$ with meas $(\mathcal{I}(T)) \ll T^{1-\delta} y(\log T)^{5}$, such that for all $t \in[T, 2 T] \backslash \mathcal{I}(T)$ and all $|s| \leq r+\delta$ we have

$$
\log \zeta\left(\frac{3}{4}+i t+s\right)=\sum_{n \leq y} \frac{\Lambda(n)}{n^{\frac{3}{4}+i t+s} \log n}+O\left(\frac{(\log y)^{2} \log T}{y^{(1 / 4-r) / 2}}\right) .
$$

To prove this result, we need the following lemma from Granville and Soundararajan [4].

Lemma 4.2 (Lemma 1 of [4]). Let $y \geq 2$ and $|t| \geq y+3$ be real numbers. Let $1 / 2 \leq \sigma_{0}<1$ and suppose that the rectangle $\left\{z: \sigma_{0}<\operatorname{Re}(z) \leq 1,|\operatorname{Im}(z)-t| \leq y+2\right\}$ is free of zeros of $\zeta(z)$. Then for any $\sigma$ with $\sigma_{0}+2 / \log y<\sigma \leq 1$ we have

$$
\log \zeta(\sigma+i t)=\sum_{n \leq y} \frac{\Lambda(n)}{n^{\sigma+i t} \log n}+O\left(\log |t| \frac{(\log y)^{2}}{y^{\sigma-\sigma_{0}}}\right) .
$$

Proof of Lemma 4.1. Let $\sigma_{0}=1 / 2+\delta$. For $j=1,2$ let $\mathcal{T}_{j}$ be the set of those $t \in[T, 2 T]$ for which the rectangle

$$
\left\{z: \sigma_{0}<\operatorname{Re}(z) \leq 1,|\operatorname{Im}(z)-t|<y+1+j\right\}
$$

is free of zeros of $\zeta(z)$. Then, note that $\mathcal{T}_{2} \subseteq \mathcal{T}_{1}$, and for all $t \in \mathcal{T}_{2}$, we have $t+\operatorname{Im}(s) \in \mathcal{T}_{1}$ for all $|s| \leq r+\delta$. Hence, by Lemma 4.2 we have

$$
\log \zeta\left(\frac{3}{4}+i t+s\right)=\sum_{n \leq y} \frac{\Lambda(n)}{n^{3 / 4+i t+s} \log n}+O\left(\frac{(\log y)^{2} \log T}{y^{(1 / 4-r) / 2}}\right),
$$

for all $t \in \mathcal{T}_{2}$ and all $|s| \leq r+\delta$. Let $N(\sigma, T)$ be the number of zeros of $\zeta(s)$ in the rectangle $\sigma<\operatorname{Re}(s) \leq 1$ and $|\operatorname{Im}(s)| \leq T$. By the classical zero density estimate $N(\sigma, T) \ll T^{3 / 2-\sigma}(\log T)^{5}$ (see for example Theorem 9.19 A ot Titchmarsh [12]) we deduce that the measure of the complement of $\mathcal{T}_{2}$ in $[T, 2 T]$ is $\ll T^{1-\delta} y(\log T)^{5}$.

We also require a minor variant of Lemma 3.3 of [10], whose proof we will omit.

Lemma 4.3. Fix $1 / 2<\sigma<1$, and let $s$ be a complex number such that $\operatorname{Re}(s)=\sigma$, and $|\operatorname{Im}(s)| \leq 1$. Then, for any positive integer $k \leq \log T /(3 \log y)$ we have

$$
\frac{1}{T} \int_{T}^{2 T}\left|\sum_{n \leq y} \frac{\Lambda(n)}{n^{s+i t} \log n}\right|^{2 k} d t \ll\left(\frac{c_{8} k^{1-\sigma}}{(\log k)^{\sigma}}\right)^{2 k}
$$


and

$$
\mathbb{E}\left(|\log \zeta(s, X)|^{2 k}\right) \ll\left(\frac{c_{8} k^{1-\sigma}}{(\log k)^{\sigma}}\right)^{2 k}
$$

for some positive constant $c_{8}$ that depends at most on $\sigma$.

Proof of Proposition 2.1. Let $\delta=e^{-V / 2}$. Taking $y=(\log T)^{5(1 / 4-r)^{-1}}$ in Lemma 4.1 gives for all $t \in[T, 2 T]$ except for a set with measure $\ll T^{1-(1 / 4-r) / 5}$ that

$$
\log \zeta\left(\frac{3}{4}+i t+s\right)=\sum_{n \leq y} \frac{\Lambda(n)}{n^{3 / 4+i t+s} \log n}+O\left(\frac{1}{\log T}\right),
$$

for all $|s| \leq r+\delta$. Furthermore, it follows from Cauchy's integral formula that

$$
\left(\sum_{n \leq y} \frac{\Lambda(n)}{n^{3 / 4+i t+s} \log n}\right)^{2 k}=\frac{1}{2 \pi i} \int_{|z|=r+2 \delta}\left(\sum_{n \leq y} \frac{\Lambda(n)}{n^{3 / 4+i t+z} \log n}\right)^{2 k} \frac{d z}{z-s} .
$$

Applying Lemma 4.3 we get that

$$
\begin{aligned}
\frac{1}{T} \int_{T}^{2 T}\left(\max _{|s| \leq r+\delta}\left|\sum_{n \leq y} \frac{\Lambda(n)}{n^{3 / 4+i t+s} \log n}\right|\right)^{2 k} d t & \ll \frac{1}{\delta} \int_{|z|=r+2 \delta} \frac{1}{T} \int_{T}^{2 T}\left|\sum_{n \leq y} \frac{\Lambda(n)}{n^{3 / 4+i t+z} \log n}\right|^{2 k} d t|d z| \\
& \ll e^{V / 2}\left(c_{8}(r) \frac{k^{1-\sigma^{\prime}(r)}}{(\log k)^{\sigma^{\prime}(r)}}\right)^{2 k}
\end{aligned}
$$

where $\sigma^{\prime}(r)=\frac{3}{4}-r-2 \delta$, and $k \leq c_{9} \log T / \log \log T$, for some sufficiently small constant $c_{9}>0$. We now choose $k=\left\lfloor c_{6}(r) V^{\frac{1}{(1-\sigma(r))}}(\log V)^{\frac{\sigma(r)}{1-\sigma(r)}}\right\rfloor\left(\right.$ so that $\left.k^{\sigma^{\prime}(r)} \asymp k^{\sigma(r)}\right)$ where $c_{6}(r)$ is a sufficiently small absolute constant. Using (4.2) and (4.3) along with Chebyshev's inequality and the above estimate we conclude that there exists $c_{7}(r)>0$ such that

$$
\begin{aligned}
\mathbb{P}_{T}\left(\max _{|z| \leq r}\left|\zeta^{\prime}\left(\frac{3}{4}+i t+z\right)\right|>e^{V}\right) & \ll \mathbb{P}_{T}\left(\max _{|s| \leq r+\delta}\left|\sum_{n \leq y} \frac{\Lambda(n)}{n^{3 / 4+i t+s} \log n}\right|>\frac{V}{4}\right)+T^{1-(1 / 4-r) / 5} \\
& \ll e^{V / 2}\left(\frac{4}{V} \cdot c_{8}(r) \frac{k^{1-\sigma^{\prime}(r)}}{(\log k)^{\sigma^{\prime}(r)}}\right)^{2 k}+T^{1-(1 / 4-r) / 5} \\
& \ll \exp \left(-c_{6} V^{\frac{1}{1-\sigma(r)}}(\log V)^{\frac{\sigma(r)}{1-\sigma(r)}}\right)
\end{aligned}
$$

for $V \leq c_{7}(\log T)^{1-\sigma(r)} / \log \log T$.

We now prove Proposition 2.2 along the same lines. The proof is in fact easier than in the zeta function case, since we can compute the moments of $\log \zeta(s, X)$, for any $s$ with $\operatorname{Re}(s)>1 / 2$. 
Proof of Proposition 2.2. Let $\delta=e^{-V / 2}$. Since $\zeta\left(\frac{3}{4}+s, X\right)$ is almost surely analytic in $|s| \leq r+2 \delta$, then by Cauchy's estimate we have almost surely that

$$
\max _{|z| \leq r}\left|\zeta^{\prime}\left(\frac{3}{4}+z, X\right)\right| \leq \frac{1}{\delta} \max _{|s| \leq r+\delta}\left|\zeta\left(\frac{3}{4}+s, X\right)\right| .
$$

Therefore, we obtain

$$
\begin{aligned}
\mathbb{P}\left(\max _{|z| \leq r}\left|\zeta^{\prime}\left(\frac{3}{4}+z, X\right)\right|>e^{V}\right) & \leq \mathbb{P}\left(\max _{|s| \leq r+\delta}\left|\zeta\left(\frac{3}{4}+s, X\right)\right|>\delta e^{V}\right) \\
& \leq \mathbb{P}\left(\max _{|s| \leq r+\delta}\left|\log \zeta\left(\frac{3}{4}+s, X\right)\right|>\frac{V}{2}\right)
\end{aligned}
$$

Let $k$ be a positive integer. By (2.2) $\log \zeta\left(\frac{3}{4}+s, X\right)$ converges almost surely to a holomorphic function in $|s| \leq r+2 \delta$. Using Cauchy's integral formula as in (4.4), we obtain almost surely that

$$
\left(\max _{|s| \leq r+\delta}\left|\log \zeta\left(\frac{3}{4}+s, X\right)\right|\right)^{2 k} \ll \frac{1}{\delta} \int_{|z|=r+2 \delta}\left|\log \zeta\left(\frac{3}{4}+z, X\right)\right|^{2 k} \cdot|d z| .
$$

Hence, applying Lemma 4.3 we get

$$
\begin{aligned}
\mathbb{P}\left(\max _{|s| \leq r+\delta}\left|\log \zeta\left(\frac{3}{4}+s, X\right)\right|>V / 2\right) & \leq\left(\frac{2}{V}\right)^{2 k} \cdot \mathbb{E}\left(\left(\max _{|s| \leq r+\delta}\left|\zeta\left(\frac{3}{4}+s, X\right)\right|\right)^{2 k}\right) \\
& \ll\left(\frac{2}{V}\right)^{2 k} e^{V / 2} \int_{|z|=r+2 \delta} \mathbb{E}\left(\left|\log \zeta\left(\frac{3}{4}+z, X\right)\right|^{2 k}\right) \cdot|d z| \\
& \ll e^{V / 2}\left(\frac{2 c_{8}(r) k^{1-\sigma^{\prime}(r)}}{V(\log k)^{\sigma^{\prime}(r)}}\right)^{2 k},
\end{aligned}
$$

where $\sigma^{\prime}(r)=\frac{3}{4}-r-2 \delta$. Let $\sigma(r)=\frac{3}{4}-r$ and take $k=\left\lfloor c_{6} V^{\frac{1}{1-\sigma(r)}}(\log V)^{\frac{\sigma(r)}{1-\sigma(r)}}\right\rfloor$, where $c_{6}$ is sufficiently small (note that $k^{\sigma^{\prime}(r)} \asymp k^{\sigma(r)}$ ), then apply (4.6) to complete the proof.

\section{Moments of Joint shifts of $\log \zeta(s)$ : Proof of Theorem 2.1}

The proof of Theorem 2.1 splits into two parts. In the first part we derive an approximation to

$$
\prod_{j=1}^{k} \log \zeta\left(s_{j}+i t\right)
$$

by a short Dirichlet polynomial. In the second part we compute the resulting meanvalues and obtain Theorem 2.1. 
5.1. Approximating $\prod_{j=1}^{k} \log \zeta\left(s_{j}+i t\right)$ by short Dirichlet polynomials. Fix $1 / 2<$ $\sigma_{0}<1$, and let $\delta:=\sigma_{0}-1 / 2$. Let $k \leq \log T$ be a positive integer and $s_{1}, s_{2}, \ldots, s_{k}$ be complex numbers (not necessarily distinct) in the rectangle $\left\{z: \sigma_{0} \leq \operatorname{Re}(z)<\right.$ 1 , and $\left.|\operatorname{Im}(z)| \leq T^{\delta / 4}\right\}$. We let $\mathbf{s}=\left(s_{1}, \ldots, s_{k}\right)$, and define

$$
F_{\mathbf{s}}(n)=\sum_{\substack{n_{1}, n_{2}, \ldots, n_{k} \geq 2 \\ n_{1} n_{2} \cdots n_{k}=n}} \prod_{\ell=1}^{k} \frac{\Lambda\left(n_{\ell}\right)}{n_{\ell}^{s_{\ell}} \log \left(n_{\ell}\right)}
$$

Then for all complex numbers $z$ with $\operatorname{Re}(z)>1-\sigma_{0}$ we have

$$
\prod_{\ell=1}^{k} \log \zeta\left(s_{\ell}+z\right)=\sum_{n=1}^{\infty} \frac{F_{\mathbf{s}}(n)}{n^{z}} .
$$

The main result of this subsection is the following proposition.

Proposition 5.1. Let $T$ be large, $s_{1}, \ldots, s_{k}$ be as above, and $\mathcal{E}(T)$ be as in Lemma 5.3 below. Then, there exist positive constants $a\left(\sigma_{0}\right), b\left(\sigma_{0}\right)$ such that if $k \leq a\left(\sigma_{0}\right)(\log T) / \log \log T$ and $t \in[T, 2 T] \backslash \mathcal{E}(T)$ then

$$
\prod_{j=1}^{k} \log \zeta\left(s_{j}+i t\right)=\sum_{n \leq T^{\delta / 8}} \frac{F_{\mathbf{s}}(n)}{n^{i t}}+O\left(T^{-b\left(\sigma_{0}\right)}\right) .
$$

This depends on a sequence of fairly standard lemmas which we now describe.

Lemma 5.1. With the same notation as above, we have

$$
\left|F_{\mathbf{s}}(n)\right| \leq \frac{(2 \log n)^{k}}{n^{\sigma}}
$$

Proof. We have

$$
\left|F_{\mathbf{s}}(n)\right| \leq \frac{1}{n^{\sigma_{0}}(\log 2)^{k}} \sum_{\substack{n_{1}, n_{2}, \ldots, n_{k} \geq 2 \\ n_{1} n_{2} \cdots n_{k}=n}} \prod_{\ell=1}^{k} \Lambda\left(n_{\ell}\right) \leq \frac{2^{k}}{n^{\sigma_{0}}}\left(\sum_{m \mid n} \Lambda(m)\right)^{k} \leq \frac{(2 \log n)^{k}}{n^{\sigma_{0}}}
$$

Lemma 5.2. Let $y \geq 2$ and $|t| \geq y+3$ be real numbers. Suppose that the rectangle $\left\{z: \sigma_{0}-\delta / 2<\operatorname{Re}(z) \leq 1,|\operatorname{Im}(z)-t| \leq y+2\right\}$ is free of zeros of $\zeta(z)$. Then, for all complex numbers $s$ such that $\operatorname{Re}(s) \geq \sigma_{0}-\delta / 4$ and $|\operatorname{Im}(s)| \leq y$ we have

$$
\log \zeta(s+i t) \ll_{\sigma_{0}} \log |t| .
$$

Proof. This follows from Theorem 9.6 B of Titchmarsh.

Lemma 5.3. Let $s_{1}, \ldots, s_{k}$ be as above. Then, there exists a set $\mathcal{E}(T) \subset[T, 2 T]$ with measure meas $(\mathcal{E}(T)) \ll T^{1-\delta / 8}$, and such that for all $t \in[T, 2 T] \backslash \mathcal{E}(T)$ we have $\zeta\left(s_{j}+\right.$ $i t+z) \neq 0$ for every $1 \leq j \leq k$ and every $z$ in the rectangle $\{z:-\delta / 2<\operatorname{Re}(z) \leq$ $\left.1,|\operatorname{Im}(z)| \leq 3 T^{\delta / 4}\right\}$. 
Proof. For every $1 \leq j \leq k$, let $\mathcal{E}_{j}(T)$ be the set of $t \in[T, 2 T]$ such that the rectangle $\left\{z:-\delta / 2<\operatorname{Re}(z) \leq 1,|\operatorname{Im}(z)| \leq 3 T^{\delta / 4}\right\}$ has a zero of $\zeta\left(s_{j}+i t+z\right)$. Then, by the classical zero density estimate $N(\sigma, T) \ll T^{3 / 2-\sigma}(\log T)^{5}$, we deduce that

$$
\operatorname{meas}\left(\mathcal{E}_{j}(T)\right) \ll T^{\delta / 4} T^{3 / 2-\sigma_{0}+\delta / 2}(\log T)^{5}<T^{1-\delta / 4}(\log T)^{5} .
$$

We take $\mathcal{E}(T)=\cup_{j=1}^{k} \mathcal{E}_{j}(T)$. Then $\mathcal{E}(T)$ satisfies the assumptions of the lemma, since $\operatorname{meas}(\mathcal{E}(T)) \ll T^{1-\delta / 4}(\log T)^{6} \ll T^{1-\delta / 8}$.

We are now ready to prove Proposition 5.1.

Proof of Proposition 5.1. Let $x=\left\lfloor T^{\delta / 8}\right\rfloor+1 / 2$. Let $c=1-\sigma_{0}+1 / \log T$, and $Y=T^{\delta / 4}$. Then by Perron's formula, we have for $t \in[T, 2 T] \backslash \mathcal{E}(T)$

$$
\frac{1}{2 \pi i} \int_{c-i Y}^{c+i Y}\left(\prod_{j=1}^{k} \log \zeta\left(s_{j}+i t+z\right)\right) \frac{x^{z}}{z} d z=\sum_{n \leq x} \frac{F_{\mathbf{s}}(n)}{n^{i t}}+O\left(\frac{x^{c}}{Y} \sum_{n=1}^{\infty} \frac{\left|F_{\mathbf{s}}(n)\right|}{n^{c}|\log (x / n)|}\right) .
$$

To bound the error term of this last estimate, we split the sum into three parts: $n \leq x / 2$, $x / 2<n<2 x$ and $n \geq 2 x$. The terms in the first and third parts satisfy $|\log (x / n)| \geq$ $\log 2$, and hence their contribution is

$$
\ll \frac{x^{1-\sigma_{0}}}{Y} \sum_{n=1}^{\infty} \frac{\left|F_{\mathbf{s}}(n)\right|}{n^{c}} \leq \frac{x^{1-\sigma_{0}}}{Y}\left(\sum_{n=1}^{\infty} \frac{\Lambda(n)}{n^{\sigma_{0}+c} \log n}\right)^{k} \leq \frac{x^{1-\sigma_{0}}(2 \log T)^{k}}{Y} \ll T^{-b\left(\sigma_{0}\right)},
$$

or some positive constant $b\left(\sigma_{0}\right)$, if $a\left(\sigma_{0}\right)$ is sufficiently small. To handle the contribution of the terms $x / 2<n<2 x$, we put $r=x-n$, and use that $|\log (x / n)| \gg|r| / x$. Then by Lemma 5.1 we deduce that the contribution of these terms is

$$
\ll \frac{x^{1-\sigma_{0}}(3 \log x)^{k}}{Y} \sum_{r \leq x} \frac{1}{r} \ll \frac{x^{1-\sigma_{0}}(3 \log x)^{k+1}}{Y} \ll T^{-b\left(\sigma_{0}\right)} .
$$

We now move the contour to the line $\operatorname{Re}(s)=-\delta / 4$. By Lemma 5.3, we do not encounter any zeros of $\zeta\left(s_{j}+i t+z\right)$ since $t \in[T, 2 T] \backslash \mathcal{E}(T)$. We pick up a simple pole at $z=0$ which leaves a residue $\prod_{j=1}^{k} \log \zeta\left(s_{j}+i t\right)$. Also Lemma 5.2 implies that for any $z$ on our contour we have

$$
\left|\log \zeta\left(s_{j}+i t+z\right)\right| \leq c\left(\sigma_{0}\right) \log T,
$$

for all $j$ where $c\left(\sigma_{0}\right)$ is a positive constant. Therefore, we deduce that

$$
\frac{1}{2 \pi i} \int_{c-i Y}^{c+i Y}\left(\prod_{j=1}^{k} \log \zeta\left(s_{j}+i t+z\right)\right) \frac{x^{z}}{z} d z=\prod_{j=1}^{k} \log \zeta\left(s_{j}+i t\right)+E_{1}
$$

where

$$
\begin{aligned}
E_{1} & =\frac{1}{2 \pi i}\left(\int_{c-i Y}^{-\delta / 4-i Y}+\int_{-\delta / 4-i Y}^{-\delta / 4+i Y}+\int_{-\delta / 4+i Y}^{c+i Y}\right)\left(\prod_{j=1}^{k} \log \zeta\left(s_{j}+i t+z\right)\right) \frac{x^{z}}{z} d z \\
& \ll \frac{x^{1-\sigma_{0}}\left(c\left(\sigma_{0}\right) \log T\right)^{k}}{Y}+x^{-\delta / 4}\left(c\left(\sigma_{0}\right) \log T\right)^{k} \log Y \ll T^{-b\left(\sigma_{0}\right)},
\end{aligned}
$$


as desired.

\subsection{An Asymptotic formula for the moment of products of shifts of $\log \zeta(s)$.}

Proof of Theorem 2.1. Let $\mathcal{E}_{1}(T)$ and $\mathcal{E}_{2}(T)$ be the corresponding exceptional sets for $\mathbf{s}$ and $\mathbf{r}$ respectively as in Lemma 5.3, and let $\mathcal{E}(T)=\mathcal{E}_{1}(T) \cup \mathcal{E}_{2}(T)$. First, note that if $t \in[T, 2 T] \backslash \mathcal{E}(T)$ then by Proposition 5.1 and Lemma 5.2 we have

$$
\left.\left|\sum_{n \leq x} \frac{F_{\mathbf{s}}(n)}{n^{i t}}\right| \ll\left(c\left(\sigma_{0}\right) \log T\right)^{k}, \text { and }\left|\sum_{m \leq x} \frac{F_{\mathbf{r}}(m)}{m^{-i t}}\right| \ll\left(c\left(\sigma_{0}\right)\right) \log T\right)^{\ell},
$$

for some positive constant $c\left(\sigma_{0}\right)$. Let $x=T^{\left(\sigma_{0}-1 / 2\right) / 8}$. Then, it follows from Proposition 5.1 that

$$
\begin{aligned}
& \frac{1}{T} \int_{[T, 2 T] \backslash \mathcal{E}(T)}\left(\prod_{j=1}^{k} \log \zeta\left(s_{j}+i t\right)\right)\left(\prod_{j=1}^{\ell} \log \zeta\left(r_{j}-i t\right)\right) d t \\
& =\frac{1}{T} \int_{[T, 2 T] \backslash \mathcal{E}(T)}\left(\sum_{n \leq x} \frac{F_{\mathbf{s}}(n)}{n^{i t}}\right)\left(\sum_{m \leq x} F_{\mathbf{r}}(m) m^{i t} d t\right) d t+O\left(T^{-b\left(\sigma_{0}\right)}(\log T)^{\max (k, \ell)}\right) \\
& =\frac{1}{T} \int_{T}^{2 T}\left(\sum_{n \leq x} \frac{F_{\mathbf{s}}(n)}{n^{i t}}\right)\left(\sum_{m \leq x} F_{\mathbf{r}}(m) m^{i t}\right) d t+O\left(T^{-b\left(\sigma_{0}\right) / 2}\right) .
\end{aligned}
$$

Furthermore, we have

$$
\frac{1}{T} \int_{T}^{2 T}\left(\sum_{n \leq x} \frac{F_{\mathbf{s}}(n)}{n^{i t}}\right)\left(\sum_{m \leq x} F_{\mathbf{r}}(n) m^{i t}\right) d t=\sum_{m, n \leq x} F_{\mathbf{s}}(n) F_{\mathbf{r}}(m) \frac{1}{T} \int_{T}^{2 T}\left(\frac{m}{n}\right)^{i t} d t
$$

The contribution of the diagonal terms $n=m$ equals $\sum_{n \leq x} F_{\mathbf{s}}(n) F_{\mathbf{r}}(n)$. On the other hand, by Lemma 5.1 the contribution of the off-diagonal terms $n \neq m$ is

$$
\ll \frac{1}{T} \sum_{\substack{m, n \leq x \\ m \neq n}} \frac{(2 \log n)^{k}(2 \log m)^{\ell}}{(m n)^{\sigma_{0}}} \frac{1}{|\log (m / n)|} \ll \frac{x^{3-2 \sigma_{0}}(2 \log x)^{k+\ell}}{T} \ll T^{-1 / 2},
$$

since $|\log (m / n)| \gg 1 / x$.

Furthermore, it follows from (2.3) that

$$
\mathbb{E}\left(\prod_{j=1}^{k} \log \zeta\left(s_{j}, X\right)\right)\left(\prod_{j=1}^{\ell} \log \overline{\zeta\left(r_{j}, X\right)}\right)=\sum_{n=1}^{\infty} F_{\mathbf{s}}(n) F_{\mathbf{r}}(n)=\sum_{n \leq x} F_{\mathbf{s}}(n) F_{\mathbf{r}}(n)+E_{2},
$$

where

$$
E_{2} \leq \sum_{n>x} \frac{(2 \log n)^{k+\ell}}{n^{2 \sigma_{0}}}
$$


Since the function $(\log t)^{\beta} / t^{\alpha}$ is decreasing for $t>\exp (\beta / \alpha)$, then with the choice $\alpha=\left(2 \sigma_{0}-1\right) / 2$ we obtain

$$
E_{2} \leq \frac{(2 \log x)^{k+\ell}}{x^{\alpha}} \sum_{n>x} \frac{1}{n^{1+\alpha}} \ll \frac{(2 \log x)^{k+\ell}}{x^{2 \alpha}} \ll x^{-\alpha}
$$

Combining this with (5.2), (5.3), and (5.4) completes the proof.

\section{The Characteristic FunCtion of JOINT Shifts of $\log \zeta(s)$}

Proof of Theorem 2.2. Let $\mathcal{E}(T)$ be as in Theorem 2.1. Let $N=[\log T /(C(\log \log T))]$ where $C$ is a suitably large constant. Then, $\Phi_{T}(\mathbf{u}, \mathbf{v})$ equals

$$
\frac{1}{T} \int_{[T, 2 T] \backslash \mathcal{E}(T)} \exp \left(i\left(\sum_{j=1}^{J}\left(u_{j} \operatorname{Re} \log \zeta\left(s_{j}+i t\right)+v_{j} \operatorname{Im} \log \zeta\left(s_{j}+i t\right)\right)\right)\right) d t+O\left(T^{-c_{3}}\right)
$$

$$
=\sum_{n=0}^{2 N-1} \frac{i^{n}}{n !} \cdot \frac{1}{T} \int_{[T, 2 T] \backslash \mathcal{E}(T)}\left(\sum_{j=1}^{J}\left(u_{j} \operatorname{Re} \log \zeta\left(s_{j}+i t\right)+v_{j} \operatorname{Im} \log \zeta\left(s_{j}+i t\right)\right)\right)^{n} d t+E_{3},
$$

where

$$
E_{3} \ll T^{-c_{3}}+\frac{1}{(2 N) !}\left(\frac{2 c_{1}(\log T)^{\sigma}}{J}\right)^{2 N} \frac{1}{T} \int_{[T, 2 T] \backslash \mathcal{E}(T)}\left(\sum_{j=1}^{J}\left|\log \zeta\left(s_{j}+i t\right)\right|\right)^{2 N} d t
$$

Now, by Theorem 2.1 along with Lemma 4.3, we obtain that for all $1 \leq j \leq J$

$$
\frac{1}{T} \int_{[T, 2 T] \backslash \mathcal{E}(T)}\left|\log \zeta\left(s_{j}+i t\right)\right|^{2 N} d t \ll \mathbb{E}\left(\left|\log \zeta\left(s_{j}, X\right)\right|^{2 N}\right) \leq\left(\frac{c_{8}(\sigma) N^{1-\sigma}}{(\log N)^{\sigma}}\right)^{2 N}
$$

for some positive constant $c_{8}=c_{8}(\sigma)$. Furthermore, by Minkowski's inequality we have

$$
\frac{1}{T} \int_{[T, 2 T] \backslash \mathcal{E}(T)}\left(\sum_{j=1}^{J}\left|\log \zeta\left(s_{j}+i t\right)\right|\right)^{2 N} d t \leq\left(c_{8} J \frac{N^{1-\sigma}}{(\log N)^{\sigma}}\right)^{2 N}
$$

Therefore, we deduce that for some positive constant $c_{9}=c_{9}(\sigma)$, we have

$$
E_{3} \ll T^{-c_{3}}+\left(c_{9} \frac{(\log T)^{\sigma}}{(N \log N)^{\sigma}}\right)^{2 N} \ll e^{-N}
$$


Next, we handle the main term of (6.1). Let $\tilde{u}_{j}=\left(u_{j}+i v_{j}\right) / 2$ and $\tilde{v}_{j}=\left(u_{j}-i v_{j}\right) / 2$. Then by Theorem 2.1 we obtain

$$
\begin{aligned}
& \frac{1}{T} \int_{[T, 2 T] \backslash \mathcal{E}(T)}\left(\sum_{j=1}^{J}\left(u_{j} \operatorname{Re} \log \zeta\left(s_{j}+i t\right)+v_{j} \operatorname{Im} \log \zeta\left(s_{j}+i t\right)\right)\right)^{n} d t \\
& \frac{1}{T} \int_{[T, 2 T] \backslash \mathcal{E}(T)}\left(\sum_{j=1}^{J}\left(\tilde{u}_{j} \log \zeta\left(s_{j}+i t\right)+\tilde{v}_{j} \log \zeta\left(s_{j}-i t\right)\right)\right)^{n} d t \\
& =\sum_{\substack{k_{1}, \ldots, k_{2 J} \geq 0 \\
k_{1}+\cdots+k_{2 J}=n}}\left(\begin{array}{c}
n \\
k_{1}, k_{2}, \ldots, k_{2 J}
\end{array}\right) \prod_{j=1}^{J} \tilde{u}_{j}^{k_{j}} \prod_{\ell=1}^{J} \tilde{v}_{\ell}^{k_{J+\ell}} \\
& \times \frac{1}{T} \int_{[T, 2 T] \backslash \mathcal{E}(T)} \prod_{j=1}^{J}\left(\log \zeta\left(s_{j}+i t\right)\right)^{k_{j}} \prod_{\ell=1}^{J}\left(\log \zeta\left(s_{\ell}-i t\right)\right)^{k_{J}+\ell} d t \\
& =\sum_{\substack{k_{1}, \ldots, k_{2 J} \geq 0 \\
k_{1}+\cdots+k_{2 J}=n}}\left(\begin{array}{c}
n \\
k_{1}, k_{2}, \ldots, k_{2 J}
\end{array}\right) \prod_{j=1}^{J} \tilde{u}_{j}^{k_{j}} \prod_{\ell=1}^{J} \tilde{v}_{\ell}^{k_{J+\ell}} \\
& \times \mathbb{E}\left(\prod_{j=1}^{J}\left(\log \zeta\left(s_{j}, X\right)\right)^{k_{j}} \prod_{\ell=1}^{J}\left(\log \zeta\left(s_{\ell}, X\right)\right)^{k_{J+\ell}}\right)+O\left(T^{-c_{5}}\left(2 c_{1}(\log T)^{\sigma}\right)^{n}\right), \\
& =\mathbb{E}\left(\left(\sum_{j=1}^{J}\left(u_{j} \operatorname{Re} \log \zeta\left(s_{j}, X\right)+v_{j} \operatorname{Im} \log \zeta\left(s_{j}, X\right)\right)\right)^{n}\right)+O\left(T^{-c_{5}}\left(2 c_{1}(\log T)^{\sigma}\right)^{n}\right) .
\end{aligned}
$$

Inserting this estimate in (6.1), we derive

$$
\begin{aligned}
\Phi_{T}(\mathbf{u}, \mathbf{v}) & =\sum_{n=0}^{2 N-1} \frac{i^{n}}{n !} \mathbb{E}\left(\left(\sum_{j=1}^{J}\left(u_{j} \operatorname{Re} \log \zeta\left(s_{j}, X\right)+v_{j} \operatorname{Im} \log \zeta\left(s_{j}, X\right)\right)\right)^{n}\right)+O\left(e^{-N}\right) \\
& =\Phi_{\text {rand }}(\mathbf{u}, \mathbf{v})+E_{4} .
\end{aligned}
$$

where

$$
E_{4} \ll e^{-N}+\frac{1}{(2 N) !}\left(\frac{2 c_{1}(\log T)^{\sigma}}{J}\right)^{2 N} \mathbb{E}\left(\left(\sum_{j=1}^{J}\left|\log \zeta\left(s_{j}, X\right)\right|\right)^{2 N}\right) \ll e^{-N}
$$

by (6.2) and Minkowski's inequality. This completes the proof.

\section{Discrepancy eStimates FOR THE Distribution OF SHIFts}

The deduction of Theorem 2.3 from Theorem 2.2 uses Beurling-Selberg functions. For $z \in \mathbb{C}$ let

$$
H(z)=\left(\frac{\sin \pi z}{\pi}\right)^{2}\left(\sum_{n=-\infty}^{\infty} \frac{\operatorname{sgn}(n)}{(z-n)^{2}}+\frac{2}{z}\right) \quad \text { and } \quad K(z)=\left(\frac{\sin \pi z}{\pi z}\right)^{2} .
$$

Beurling proved that the function $B^{+}(x)=H(x)+K(x)$ majorizes $\operatorname{sgn}(x)$ and its Fourier transform has restricted support in $(-1,1)$. Similarly, the function $B^{-}(x)=$ 
$H(x)-K(x)$ minorizes $\operatorname{sgn}(x)$ and its Fourier transform has the same property (see Vaaler [13] Lemma 5).

Let $\Delta>0$ and $a, b$ be real numbers with $a<b$. Take $\mathcal{I}=[a, b]$ and define

$$
F_{\mathcal{I}}(z)=\frac{1}{2}\left(B^{-}(\Delta(z-a))+B^{-}(\Delta(b-z))\right) .
$$

The function $F_{\mathcal{I}}$ has the following remarkable properties. First, it follows from the inequality $B^{-}(x) \leq \operatorname{sgn}(x) \leq B^{+}(x)$ that

$$
0 \leq \mathbf{1}_{\mathcal{I}}(x)-F_{\mathcal{I}}(x) \leq K(\Delta(x-a))+K(\Delta(b-x)) .
$$

Additionally, one has

$$
\widehat{F}_{\mathcal{I}}(\xi)=\left\{\begin{array}{l}
\widehat{\mathbf{1}}_{\mathcal{I}}(\xi)+O\left(\frac{1}{\Delta}\right) \text { if }|\xi|<\Delta, \\
0 \text { if }|\xi| \geq \Delta .
\end{array}\right.
$$

The first estimate above follows from (7.1) and the second follows from the fact that the Fourier transform of $B^{-}$is supported in $(-1,1)$. Before proving Theorem 2.3 we first require the following lemmas.

Lemma 7.1. For $x \in \mathbb{R}$ we have $\left|F_{\mathcal{I}}(x)\right| \leq 1$.

Proof. It suffices to prove the lemma for $\Delta=1$. Also, note that we only need to show that $F_{\mathcal{I}}(x) \geq-1$. From the identity

$$
\sum_{n=-\infty}^{\infty} \frac{1}{(n-z)^{2}}=\left(\frac{\pi}{\sin \pi z}\right)^{2}
$$

it follows that for $y \geq 0$

$$
H(y)=1-K(y) G(y)
$$

where

$$
G(y)=2 y^{2} \sum_{m=0}^{\infty} \frac{1}{(y+m)^{2}}-2 y-1 .
$$

In Lemma 5 of [13], Vaaler shows for $y \geq 0$ that

$$
0 \leq G(y) \leq 1
$$

Also, note that for each $m \geq 1$, and $0<y \leq 1$ one has $\frac{m}{(y+m)^{3}} \leq \int_{m-1}^{m} \frac{t}{(y+t)^{3}} d t$ so that for $0<y \leq 1$

$$
G^{\prime}(y)=4 y \sum_{m \geq 1} \frac{m}{(y+m)^{3}}-2 \leq 4 y \int_{0}^{\infty} \frac{t}{(y+t)^{3}} d t-2=0 .
$$

First consider the case $a \leq x \leq b$. By (7.3) we get that in this range

$$
F_{\mathcal{I}}(x)=\frac{1}{2}(2-K(x-a)(G(x-a)+1)-K(b-x)(G(b-x)+1)),
$$


which along with (7.4) implies $F_{\mathcal{I}}(x) \geq-1$ for $a \leq x \leq b$. Now consider the case $x<a$. Since $H$ is an odd function (7.3) and (7.4) imply

$$
\begin{aligned}
F_{\mathcal{I}}(x) & =\frac{1}{2}(K(x-a)(G(a-x)-1)-K(b-x)(G(b-x)+1)) \\
& \geq \frac{1}{2}(-K(x-a)-2 K(x-b)),
\end{aligned}
$$

which is $\geq-1$ if $K(x-b) \leq 1 / 2$. If $K(x-b) \geq 1 / 2$ we also have $K(x-a)>K(x-b)$ and $0<b-x<1$. By this and (7.5) we have in this range as well that

$$
F_{\mathcal{I}}(x) \geq \frac{1}{2}(K(x-b)(G(a-x)-G(b-x)-2)) \geq-1 .
$$

Hence, $F_{\mathcal{I}}(x) \geq-1$ for $x<a$. The remaining case when $x>b$ follows from a similar argument.

Lemma 7.2. Fix $1 / 2<\sigma<1$, and let $s$ be a complex number such that $\operatorname{Re}(s)=\sigma$ and $|\operatorname{Im}(s)| \leq T^{\frac{1}{4} \cdot\left(\sigma-\frac{1}{2}\right)}$. Then there exists a positive constant $c_{1}(\sigma)$ such that for $|u| \leq$ $c_{1}(\sigma)(\log T)^{\sigma}$ we have

$$
\Phi_{T}(u, 0) \ll \exp \left(\frac{-u}{5 \log u}\right) \quad \text { and } \quad \Phi_{T}(0, u) \ll \exp \left(\frac{-u}{5 \log u}\right) .
$$

Proof. By a straightforward modification of Lemma 6.3 of [10] one has that

$$
\mathbb{E}(\exp (i u \operatorname{Re} \log \zeta(s, X))) \ll \exp \left(-\frac{u}{5 \log u}\right)
$$

and

$$
\mathbb{E}(\exp (i u \operatorname{Im} \log \zeta(s, X))) \ll \exp \left(-\frac{u}{5 \log u}\right) .
$$

Using the first bound and applying Theorem 2.2 with $J=1$ establishes the first claim. The second claim follows similarly by using the second bound and Theorem 2.2 ,

Proof of Theorem 2.3. First, we claim that it suffices to estimate the discrepancy over $\left(\mathcal{R}_{1}, \ldots, \mathcal{R}_{J}\right)$ such that for each $j$ we have $\mathcal{R}_{j} \subset[-\sqrt{\log T}, \sqrt{\log T}] \times[-\sqrt{\log T}, \sqrt{\log T}]$. To see this consider $\left(\widetilde{\mathcal{R}_{1}}, \ldots, \widetilde{\mathcal{R}_{J}}\right)$ where $\widetilde{\mathcal{R}_{j}}=\mathcal{R}_{j} \cap[-\sqrt{\log T}, \sqrt{\log T}] \times[-\sqrt{\log T}, \sqrt{\log T}]$. It follows that

$$
\begin{aligned}
& \left|\mathbb{P}_{T}\left(\log \zeta\left(s_{j}+i t\right) \in \mathcal{R}_{j}, \forall j \leq J\right)-\mathbb{P}_{T}\left(\log \zeta\left(s_{1}+i t\right) \in \widetilde{\mathcal{R}_{1}}, \log \zeta\left(s_{j}+i t\right) \in \mathcal{R}_{j}, 2 \leq j \leq J\right)\right| \\
& \ll \mathbb{P}_{T}\left(\left|\log \zeta\left(s_{1}+i t\right)\right| \geq \sqrt{\log T}\right) \ll \exp (-\sqrt{\log T}),
\end{aligned}
$$

where the last bound follows from Theorem 1.1 and Remark 1 of of [9]. Repeating this argument gives

$$
\left|\mathbb{P}_{T}\left(\log \zeta\left(s_{j}+i t\right) \in \widetilde{\mathcal{R}_{j}}, \forall j \leq J\right)-\mathbb{P}_{T}\left(\log \zeta\left(s_{j}+i t\right) \in \mathcal{R}_{j}, \forall j \leq J\right)\right| \ll J \exp (-\sqrt{\log T}) .
$$


Similarly,

$\left|\mathbb{P}\left(\log \zeta\left(s_{j}, X\right) \in \widetilde{\mathcal{R}_{j}}, \forall j \leq J\right)-\mathbb{P}\left(\log \zeta\left(s_{j}, X\right) \in \mathcal{R}_{j}, \forall j \leq J\right)\right| \ll J \exp (-\sqrt{\log T})$.

Hence, the error from restricting to $\left(\widetilde{\mathcal{R}_{1}}, \ldots, \widetilde{\mathcal{R}_{J}}\right)$ is negligible and establishes the claim.

Let $\Delta=c_{1}(\sigma)(\log T)^{\sigma} / J$ and $\mathcal{R}_{j}=\left[a_{j}, b_{j}\right] \times\left[c_{j}, d_{j}\right]$ for $j=1, \ldots, J$, with $\mid b_{j}-$ $a_{j}|,| d_{j}-c_{j} \mid \leq 2 \sqrt{\log T}$. Also, write $\mathcal{I}_{j}=\left[a_{j}, b_{j}\right]$ and $\mathcal{J}_{j}=\left[c_{j}, d_{j}\right]$. By Fourier inversion, (7.2), and Theorem 2.2 we have that

$$
\begin{aligned}
& \frac{1}{T} \int_{T}^{2 T} \prod_{j=1}^{J} F_{\mathcal{I}_{j}}\left(\operatorname{Re} \log \zeta\left(s_{j}+i t\right)\right) F_{\mathcal{J}_{j}}\left(\operatorname{Im} \log \zeta\left(s_{j}+i t\right)\right) d t \\
= & \int_{\mathbb{R}^{2 J}}\left(\prod_{j=1}^{J} \widehat{F}_{\mathcal{I}_{j}}\left(u_{j}\right) \widehat{F}_{\mathcal{J}_{j}}\left(v_{j}\right)\right) \Phi_{T}(\mathbf{u}, \mathbf{v}) d \mathbf{u} d \mathbf{v} \\
= & \int_{\substack{\left|u_{j}\right|,\left|v_{j}\right| \leq \Delta \\
j=1,2, \ldots, J}}\left(\prod_{j=1}^{J} \widehat{F}_{\mathcal{I}_{j}}\left(u_{j}\right) \widehat{F}_{\mathcal{J}_{j}}\left(v_{j}\right)\right) \Phi_{\text {rand }}(\mathbf{u}, \mathbf{v}) d \mathbf{u} d \mathbf{v}+O\left((2 \Delta \sqrt{\log T})^{2 J} \exp \left(-\frac{c_{2} \log T}{\log \log T}\right)\right) \\
= & \mathbb{E}\left(\prod_{\substack{j=1 \\
F_{\mathcal{I}_{j}}}}\left(\operatorname{Re} \log \zeta\left(s_{j}, X\right)\right) F_{\mathcal{J}_{j}}\left(\operatorname{Im} \log \zeta\left(s_{j}, X\right)\right)\right)+O\left(\exp \left(-\frac{c_{2} \log T}{2 \log \log T}\right)\right)
\end{aligned}
$$

Next note that $\widehat{K}(\xi)=\max (0,1-|\xi|)$. Applying Fourier inversion, Theorem 2.2 with $J=1$, and Lemma 7.2 we have that

$$
\frac{1}{T} \int_{T}^{2 T} K(\Delta \cdot(\operatorname{Re} \log \zeta(s+i t)-\alpha)) d t=\frac{1}{\Delta} \int_{-\Delta}^{\Delta}\left(1-\frac{|\xi|}{\Delta}\right) e^{-2 \pi i \alpha \xi} \Phi_{T}(\xi, 0) d \xi \ll \frac{1}{\Delta}
$$

where $\alpha$ is an arbitrary real number and $s \in \mathbb{C}$ satisfies $\sigma \leq \operatorname{Re}(\mathrm{s})<1$ and $|\operatorname{Im}(s)|<$ $T^{\frac{1}{4}\left(\sigma-\frac{1}{2}\right)}$. By this and (7.1) we get that

$$
\frac{1}{T} \int_{T}^{2 T} F_{\mathcal{I}_{1}}\left(\operatorname{Re} \log \zeta\left(s_{1}+i t\right)\right) d t=\frac{1}{T} \int_{T}^{2 T} \mathbf{1}_{\mathcal{I}_{1}}\left(\operatorname{Re} \log \zeta\left(s_{1}+i t\right)\right) d t+O(1 / \Delta)
$$

Lemma 7.1 implies that $\left|F_{\mathcal{I}_{j}}(x)\right|,\left|F_{\mathcal{J}_{j}}(x)\right| \leq 1$ for $j=1, \ldots, J$. Hence, by this and (7.7)

$$
\begin{aligned}
\frac{1}{T} \int_{T}^{2 T} \prod_{j=1}^{J} F_{\mathcal{I}_{j}} & \left(\operatorname{Re} \log \zeta\left(s_{j}+i t\right)\right) F_{\mathcal{J}_{j}}\left(\operatorname{Im} \log \zeta\left(s_{j}+i t\right)\right) d t \\
=\frac{1}{T} \int_{T}^{2 T} \mathbf{1}_{\mathcal{I}_{1}}( & \left.\operatorname{Re} \log \zeta\left(s_{j}+i t\right)\right) F_{\mathcal{J}_{1}}\left(\operatorname{Im} \log \zeta\left(s_{j}+i t\right)\right) \\
& \times \prod_{j=2}^{J} F_{\mathcal{I}_{j}}\left(\operatorname{Re} \log \zeta\left(s_{j}+i t\right)\right) F_{\mathcal{J}_{j}}\left(\operatorname{Im} \log \zeta\left(s_{j}+i t\right)\right) d t+O(1 / \Delta) .
\end{aligned}
$$


Iterating this argument and using an analog of (7.7) for $\operatorname{Im} \log \zeta(s+i t)$, which is proved in the same way, gives

$$
\begin{aligned}
\frac{1}{T} \int_{T}^{2 T} \prod_{j=1}^{J} F_{\mathcal{I}_{j}} & \left(\operatorname{Re} \log \zeta\left(s_{j}+i t\right)\right) F_{\mathcal{J}_{j}}\left(\operatorname{Im} \log \zeta\left(s_{j}+i t\right)\right) d t \\
& =\mathbb{P}_{T}\left(\log \zeta\left(s_{j}+i t\right) \in \mathcal{R}_{j}, \forall j \leq J\right)+O\left(\frac{J}{\Delta}\right)
\end{aligned}
$$

Similarly, it can be shown that

$$
\begin{aligned}
\mathbb{E}\left(\prod_{j=1}^{J} F_{\mathcal{I}_{j}}\left(\operatorname{Re} \log \zeta\left(s_{j}, X\right)\right) F_{\mathcal{J}_{j}}\left(\operatorname{Im} \log \zeta\left(s_{j}, X\right)\right)\right)= & \mathbb{P}\left(\log \zeta\left(s_{j}, X\right) \in \mathcal{R}_{j}, \forall j \leq J\right) \\
& +O\left(\frac{J}{\Delta}\right) .
\end{aligned}
$$

Using (7.8) and (7.9) in (7.6) completes the proof.

\section{REFERENCES}

[1] B. Bagchi, Statistical behaviour and universality properties of the Riemann zeta-function and other allied Dirichlet series, PhD thesis, Indian Statistical Institute, Kolkota, 1981

[2] R. Garunkstis, The effective universality theorem for the Riemann zeta-function, Proceedings of the Session in Analytic Number Theory and Diophantine Equations, Bonner Math. Schriften, 360, Univ. Bonn, Bonn, 2003

[3] A. Good, On the distribution of the values of Riemann's zeta function., Acta. Arith. 38 (1980), no. 4, 347-388

[4] A. Granville and K. Soundararajan, The distribution of values of $L\left(1, \chi_{d}\right)$. Geom. Funct. Anal. 13 (2003), no. 5, 992-1028.

[5] B. Jessen, and A. Wintner, Distribution functions and the Riemann zeta function. Trans. Amer. Math. Soc. 38 (1935), no. 1, 48-88.

[6] J. Kaczorowski and A. Perelli, The Selberg class: a survey, Number theory in progress, Vol. 2 (Zakopane-Kościelisko, 1997), 953-992, de Gruyter, Berlin, 1999.

[7] E. Kowalski, Bagchi's theorem for families of automomorphic forms, pre-print.

[8] E. Kowalski and P. Michel, Zeros of families of automorphic L-functions close to 1, Pacific J. Math. 207 (2002) 411-431.

[9] Y. Lamzouri, On the distribution of extreme values of zeta and L-functions in the strip $1 / 2<\sigma<1$. Int. Math. Res. Not. IMRN 2011, no. 23, 5449-5503.

[10] Y. Lamzouri, S. Lester, and M. Radziwiłl, Discrepancy bounds for the distribution of the Riemann zeta-function and applications. arXiv:1402.6682.

[11] K. Matsumoto, A survey on the theory of universality for zeta and L-functions, Number Theory, 95-144, Ser. Number Theory Appl., 11, World Sci. Publ., Hackensack, NJ, 2015

[12] E. C. Titchmarsh, The theory of the Riemann zeta-function. Second edition. Edited and with a preface by D. R. Heath-Brown. The Clarendon Press, Oxford University Press, New York, 1986. $\mathrm{x}+412 \mathrm{pp}$.

[13] J. Vaaler, Some extremal functions in Fourier analysis. Bull Amer. Math. Soc. (N.S.) 12 (1985), no. 2, 183-216.

[14] S. M. Voronin, A theorem on the distribution of values of the Riemann zeta-function. Dokl. Akad. Nauk SSSR, 221 (1975), no. 4, 771. 
Department of Mathematics and Statistics, York University, 4700 Keele Street, Toronto, ON, M3J1P3 CANADA

E-mail address: lamzouri@mathstat.yorku.ca

Department of Mathematics and Statistics, University of Montreal Pavillon AndréAisenstadt, PO Box 6128, Centre-Ville Station Montreal, Quebec H3C 3J7

E-mail address: sjlester@gmail.com

Department of Mathematics McGill University 805 Sherbrooke Street West MonTREAL, QUEBEC H3A 0G4

E-mail address: maksym.radziwill@gmail.com 